\title{
Saúde mental no Programa de Saúde da Família: conceitos dos agentes comunitários sobre o transtorno mental
}

\author{
MENTAL HEALTH IN THE FAMILY HEALTH PROGRAM: \\ THE CONCEPTIONS OF COMMUNITY AGENTS ABOUT MENTAL ILLNESS
}

\section{SALUD MENTAL EN EL PROGRAMA DE SALUD DE LA FAMILIA: CONCEPTOS DE LOS AGENTES COMUNITARIOS SOBRE EL TRASTORNO MENTAL}

\author{
Maria Alice Ornellas Pereira', Liliane Barbieri", \\ Virginia Praciano de Paula"I', Melissa dos Santos Paschoalato Franco'v
}

\section{RESUMO}

Este estudo objetivou identificar as representações sociais de agentes comunitários de uma unidade de Programa Saúde da Família sobre o transtorno mental. Optamos pela pesquisa qualitativa, utilizando o estudo de caso. Para a coleta de dados, recorremos à entrevista semi-estruturada, enriquecida pelo uso de Técnica Projetiva, e à análise temática para analisar o material obtido. Os resultados evidenciam representações sociais ancoradas no paradigma psiquiátrico tradicional. Esse considera a pessoa acometida pelo transtorno mental passiva, sem condições de protagonizar os próprios caminhos que, por sua vez, são marcados pelo preconceito. Desse modo, denota-se a grande necessidade de investimento na capacitação em saúde mental, junto aos atores do cenário da assistência do Programa de Saúde da Família. De acordo com o estudo, tal investimento contribuirá para a efetivação de práticas e construção de novos saberes, contribuindo para a melhoria da assistência em saúde.

\section{DESCRITORES}

Pessoal de saúde.

Programa Saúde da Família.

Saúde mental.

Serviços de Saúde Mental.

\section{ABSTRACT}

This study identified social representations of mental illness by community agents in a Family Health Program unit. The authors chose the qualitative approach and used the case study methodology. Data were collected by means of semi-structured interviews, with the use of Projective and Thematic Analysis techniques. The results show social representations based on the traditional psychiatric paradigm, which considers the mentally ill as a passive person, without the necessary conditions to become the protagonist of his/her own history. This prejudice is typical of the traditional view. Therefore, the authors suggest that a substantial effort should be made to provide a better professional formation to community agents in the Family Health Program. Such an effort would propitiate improvements on practices and the construction of new knowledge in the context of community mental health assistance.

\section{KEY WORDS}

Health personnel.

Family Health Program.

Mental health.

Mental Health Services.

\section{RESUMEN}

Este estudio tuvo como objetivo identificar las representaciones sociales de agentes comunitarios de una unidad de Programa Salud de la Familia sobre el trastorno mental. Optamos por la pesquisa cualitativa, utilizando el estudio de caso. Para la colecta de datos, recorrimos a la entrevista semi-estructurada, enriquecida por el uso de la Técnica Proyectiva, y la análisis temática para analizar el material obtenido. Los resultados evidencian representaciones sociales ancoradas en el paradigma psiquiátrico tradicional. Ese considera a la persona acometida por el trastorno mental pasiva, sin condiciones de protagonizar los propios caminos que, a su vez, son marcados por el prejuicio. De ese modo, se denota la gran necesidad de inversión en la capitación en salud mental, junto a los autores del escenario de la asistencia del Programa de Salud de la Familia. De acuerdo con el estudio, tal inversión contribuirá para hacerse efectivo las prácticas y construcción de nuevos conocimientos, contribuyendo para la mejoría de la asistencia en salud.

\section{DESCRIPTORES}

Personal de salud.

Programa Salud de la Familia.

Salud mental.

Servicios de Salud Mental.
Professora Adjunta do Departamento de Enfermagem da Faculdade de Medicina de Botucatu, Universidade Estadual Paulista (UNESP). Botucatu, SP, Brasil. malice@fmb.unesp.br

II Graduanda do Curso de Enfermagem da Faculdade de Medicina de Botucatu, Universidade Estadual Paulista (UNESP) Botucatu, SP, Brasil. licabarbi@yahoo. unesp.br

III Graduanda do Curso de Enfermagem da Faculdade de Medicina de Botucatu, Universidade Estadual Paulista (UNESP). Botucatu, SP, Brasil. Vil3enf@uol.com.br

IV Mestranda do Departamento de Enfermagem Psiquiátrica e Ciências Humanas da Escola de Enfermagem de Ribeirão Preto, Universidade de São Paulo (EERP). Professora do Curso de Graduação em Enfermagem do Centro Universitário Hermínio Ometto (UNIARARAS).Ribeirão Preto, SP, Brasil. melissapaschoalato@ bol.com.br 


\section{INTRODUÇÃO}

Com a implantação da Reforma Psiquiátrica no país e, com ela, a reinserção do paciente psiquiátrico na família e na comunidade, o núcleo familiar vem, pouco a pouco, sendo considerado como unidade básica no processo de assistência. A família é responsável, na maioria das vezes, pelo apoio físico, emocional, social e psicológico ao seu familiar doente $^{(1)}$. Para cumprir seu papel de provedora, necessita, além dos recursos institucionais, do preparo e do apoio de profissionais que a orientem, tendo em vista os encargos advindos da convivência com a pessoa em situação de sofrimento psíquico ${ }^{(2)}$.

O transtorno mental traz às famílias intenso sofrimento, exigindo-lhes uma re-elaboração de sua dinâmica e estrutu$\mathrm{ra}^{(3)}$. Tal quadro é agravado pela existência de crenças inadequadas acerca dos transtornos mentais, repercutindo no relacionamento familiar e também influenciando o próprio processo de assistência prestada ${ }^{(4)}$. O modelo utilizado pelos profissionais de saúde, e o modo de convívio com a pessoa portadora de transtorno mental contribuem para as dinâmicas familiares, principalmente quando se adotam estratégias transformadoras. Estas visam construir um novo padrão de assistência em saúde mental, rompendo com modelo clássico da psiquiatria, em que o manicômio era a única resposta ao sofrimento psíquico ${ }^{(5)}$.

Há quase uma década, através do Programa de Saúde da Família (PSF), o Brasil vem adotando o modelo de assistência domiciliar, considerado estratégia importante para o cuidado em saúde, uma vez que as pessoas passam a ser atendidas em suas singulares necessidades e não por demanda ${ }^{(6)}$. Essa estratégia de cuidado requer um olhar plural frente à complexidade dos cotidianos nos diferentes contextos, e solicita um pensamento sistêmico, já que o foco de cuidado é pautado pela consideração da maneira como a família funciona e se relaciona, pontos fundamentais no processo de atendimento.

Entre tantas características do PSF, é marcante a possibilidade de se conhecer a estrutura ou sistema em que se organiza a vida de determinada comunidade; prestar uma assistência universalizada, com descentralização de decisões; e contar com bases territoriais definidas para a atuação, vendo a família e seu espaço social como núcleo básico de abordagem no atendimento à saúde ${ }^{(7)}$. Nesse sentido evidenciase a importância de

desenvolver propostas de cuidado que incluam a Saúde Mental, partindo das situações observadas e priorizadas em seu cotidiano profissional e do conhecimento dos recursos disponíveis para que estes sejam utilizados de modo efetivo, o que é extremamente importante para o alcance de seus objetivos ${ }^{(3)}$.
O PSF vem se mostrando como instrumento do processo de transformação da assistência em saúde mental, uma vez que possibilita maior aproximação entre usuário, família e profissionais, toda a comunidade enfim. Essa proximidade é um importante recurso, principalmente no que se refere ao enfrentamento dos agravos vinculados ao sofrimento psíquico, que vêm se mostrando freqüentes, e ainda pouco tratados $^{(8)}$. No entanto, estando o PSF em desenvolvimento, a saúde mental tem sido pouco contemplada nos programas de capacitação, o que muitas vezes dificulta a efetivação da assistência que tem o domicílio como espaço terapêutico.

O imaginário construído acerca dos transtornos mentais é muitas vezes constituído de representações pautadas pelo distanciamento, exclusão, periculosidade e determinismo, que vêm influenciar posturas, delinear percursos e determinar a assistência prestada nessa área ${ }^{(9)}$. A construção desse novo modelo de assistência solicita dos profissionais, ou da equipe de atendimento, disponibilidade para rever os próprios conceitos, envolvimento, compromisso e flexibilidade, pois os desafios advindos do enfrentamento da problemática ligada à experiência de aproximação com os transtornos mentais pedem, cotidianamente, uma reflexão direcionada a um movimento interno de desconstrução de princípios fortemente instituídos ${ }^{(4)}$.

Nessa perspectiva, entendemos que a equipe que presta atendimento no PSF tem um papel central na construção de um novo modelo de cuidado, em particular na área de saúde mental. Sendo o agente comunitário morador da região, vivencia situações semelhantes aos usuários da comunidade, e identifica-se com as condições de vida dos moradores, favorecendo a abordagem e interação com eles ${ }^{(10)}$. Por outro lado, a exposição dos agentes aos problemas de várias ordens vividos pela comunidade pode ocasionar sua vulnerabilidade psíquica, pois o agente é o elemento que faz parte da comunidade ao mesmo tempo em que também representa o serviço de saú$\mathrm{de}^{(11)}$. Assim, considerando a relevância do agente comunitário no interior dessa equipe, como o grande descobridor dos recursos escondidos da comunidade ${ }^{(12)}$, elaboramos nosso objetivo: identificar as representações sociais sobre o transtorno mental, por parte dos agentes comunitários de uma Unidade de Saúde da Família.

\section{MÉTODO}

Este estudo se insere nos pressupostos dos métodos qualitativos de investigação. Foi realizado durante o primeiro semestre de 2004, junto a uma Unidade de Programa de Saúde da Família (PSF) de um município do interior de São Paulo. Os sujeitos da investigação foram seis agentes comunitários dessa Unidade. A participação foi voluntária, medi- 
ante leitura e assinatura do Termo de Consentimento Livre e Esclarecido, conforme aprovação em Comitê de Ética (Of. 278.2004-CEP, de 07 de junho de 2004). Tivemos a anuência dos seis agentes comunitários que ali desenvolviam suas atividades.

Para a coleta de dados, utilizamos a entrevista semiestruturada com cada um dos sujeitos, nas dependências da Unidade do PSF. Mediante a autorização de cada um, empregamos um gravador para o registro das falas e, como instrumento auxiliar, o Procedimento Apresentativo-Expressivo ${ }^{(13)}$. Esse procedimento consiste em uma técnica encoberta de pesquisa, por possibilitar a expressão de conteúdos subjacentes. Além dos dados de caracterização do sujeito e da questão norteadora (Como você pensa o transtorno mental?), solicitamos de cada agente um desenho que representasse sua visão individual do transtorno mental, um nome para o desenho, e a elaboração de uma estória daquela produção gráfica.

Na fase de análise, após a transcrição das entrevistas, procedemos à leitura do material obtido, utilizando a Atenção Flutuante ${ }^{(14)}$. Em seguida, recorremos à Análise Temática ${ }^{(15)}$, enfocando o conjunto das entrevistas, através de uma rede de categorias projetadas sobre os conteúdos, apreendendo os temas emergentes.

Na fase posterior, utilizamos a teoria das Representações Sociais ${ }^{(16)}$. Considera-se que estas representações se origi-nem de relações interpessoais, sendo que, uma vez constituídas, passam a influir na conduta dos indivíduos. Nesta ótica, as representações são construídas a partir de conceitos, afirmações e explicações presentes no meio social, e expressam a interação constante entre o indivíduo e a sociedade. A análise dos dados obtidos na investigação buscou identificar as representações acerca dos transtornos mentais trazidas pelos sujeitos ouvidos.

\section{APRESENTAÇÃOE DISCUSSÃO DOS RESULTADOS}

Foram ouvidos seis sujeitos, sendo quatro mulheres e dois homens, com idades variando entre vinte e um e trinta e quatro anos. Quanto à escolaridade, um sujeito possui ensino médio superior completo e um, incompleto; e quatro possuem ensino médio completo. O tempo de serviço no PSF variou de cinco a quatorze meses.

As narrativas individuais dos sujeitos permitiram identificar suas representações acerca do transtorno mental. Há um tema comum em todos os discursos: a idéia de que o transtorno mental traz segregação e exclusão. Outro aspecto comum foi o pensamento do transtorno mental como situação imutável, marcada pela fixidez de um estado de ser.

Assim, o material obtido nos permitiu apreender:

\section{a) Representação do Transtorno Mental como Loucura:}

Nos relatos, evidencia-se a noção de loucura como expressão da pessoa que não sabe o que faz, isto é, em situação de irracionalidade permanente:

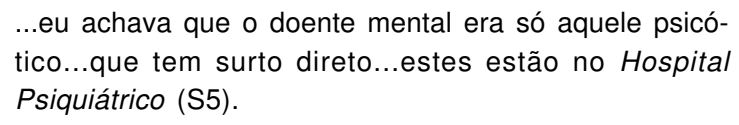

Segundo esse sujeito, para tal situação, o manicômio é o local destinado até então, para as pessoas que tem surto direto.

O exposto sugere que o hospital psiquiátrico é visto como o protetor que mantém afastado o quadro indesejado de loucura permanente, concepção que dá ancoragem à psiquiatria tradicional que, por longo período, teve no manicômio seu principal instrumento de ação. Como conseqüência dessa proteção, deixou uma herança de vidas cerceadas e excluídas de qualquer tipo de convívio e de possibilidades sociais, afetivas, familiares, ignorando-lhes os atributos residuais humanos, como habilidade, capacidade, desejos etc.

Essas práticas psiquiátricas vieram embasadas num discurso científico e submetidas, portanto, à linguagem dos especialistas na área. A representação social do transtorno mental entre profissionais (médicos) que participaram de uma pesquisa em uma capital do nordeste brasileiro é que o hospital psiquiátrico constitui-se no local apropriado para o tratamento ${ }^{(17)}$. Essa ancoragem socializa uma representação de transtorno mental entre os profissionais da área, e projeta para o mundo, via poder e hegemonia médica, a condição de se viver constantemente sob o signo da identidade entre loucura e transtorno mental.

\begin{abstract}
Aquilo que uma sociedade concebe e imagina sobre determinado objeto social, sustenta e legitima as práticas sociais tidas como corretas em relação a ele. Assim, os mitos de pensamento e representação, os mitos e as crenças presentes no imaginário social acerca da loucura, são diretamente responsáveis pelas normas de conduta prescritas pela sociedade para a identificação e recuperação das pessoas loucas ${ }^{(18)}$.
\end{abstract}

O relacionamento interpessoal estabelecido e o cuidado prestado ao portador de transtorno mental têm ligação direta com as concepções ou representações do transtorno mental $^{(19)}$.

Assim, as condutas, as manifestações incompreensíveis, as funções alteradas e as dificuldades de comunicação, dependendo da visão de quem assiste, consistem-se em referenciais que podem ser decisórios no processo de evolução do transtorno vivido pelo paciente.

$\mathrm{Na}$ produção gráfica do desenho da representação do transtorno mental aparece o título rasgando dinheiro e a elaboração da estória: 
o doente seria um louco rasgando dinheiro... é uma pessoa sem noção do certo e do errado, sem controle emocional nem social (S3).

A narrativa demonstra a visão estereotipada do louco sem trégua, desrazonado e sem limites, a necessitar, portanto, de maior proteção, conforme evidenciado na produção do desenho de título Casa de Repouso ou Hospital (S6). Isso confirma, mais uma vez, que o olhar do profissional ajuda na determinação do atendimento prestado.

Os conceitos sobre transtornos mentais, pesquisados em uma equipe de enfermagem de um hospital geral, confirmam que a conduta da assistência psiquiátrica ainda é pautada pela hegemonia médica e exclusão ${ }^{(20)}$. Assim, essa hegemonia contribui para a legitimação de práticas, incorpora saberes e representações que circulam no cotidiano, o que é evidenciado no desenho de um cadeado e uma corrente, com o título: preconceito, com a estória:

... a sociedade prende o doente mental dentro de seu mundo com medo que o doente afete o mundo da sociedade1 (S1).

Grande parte das pessoas que permaneceram por longos períodos nos manicômios, longe do convívio com a sociedade, hoje se encontra em suas comunidades, porém a exclusão ainda ronda suas vidas, elas permanecem isoladas, sem acesso às informações, lazer, saúde ou trabaIho; portanto, estar na comunidade não significa melhoria da condição de vida, e o sofrimento permanece. Portanto é necessária uma desconstrução de manicômios presentes no interior de cada profissional, assim como no próprio sistema, como um todo(4).

\section{b) Representação de Transtorno Mental como Incapacidade e Impossibilidade de Autonomia}

A autonomia pode ser entendida como condição de uma pessoa para produzir normas, gerar ordens, regras para sua vida, conforme as diferentes situações que venha a enfren$\operatorname{tar}^{(21)}$. No entanto, os discursos demonstram a idéia de que a pessoa com transtorno mental sofre também uma impossibilidade de emancipação e de auto-gerenciamento da própria vida:

... você tem que dar mais atenção para ele ter um acompanhamento mais rigoroso, você não pode deixar essa pessoa em casa sozinho... o cuidado como você dá para uma criança (S6).

Tal crença pode conduzir à falência social, isto é, no percurso de dessocialização há um déficit de competência pessoal e social. O processo de cronificação seria como um distanciamento recíproco entre o paciente e os outros. $\mathrm{Na}$ sequiência desse processo estão presentes as frustrações e os desapontamentos diante das várias falências da pessoa, levando-a auto-isolamento, ao distanciamento das pessoas significativas e ao distanciamento social. Essa seqüência conduz à espiral viciada rumo à estabilização crônica $^{(22)}$.
A representação elaborada por nossos sujeitos com relação ao transtorno mental enquanto ausência de autonomia traduz a própria mitificação que envolve os transtornos mentais, facilitando a permanência do conceito de estado de cronicidade, fixando a pessoa portadora de sofrimento psíquico em um estado de loucura subjetivado, a um modo de ser permanente ou imutável.

Desse modo, o transtorno mental pensado como obstrução às possibilidades de desenvolvimento ou realização de desejos, de expansão, de descobertas, é explicitado:

... os doentes mentais vivem presos no seu mundo por causa do seu problema.... sempre tendo que lutar contra esse problema (S2).

É possível visualizar algum rompimento para essa prisão? A idéia de aprisionamento mencionada no discurso dificulta vislumbrar o futuro, como se houvesse certa desistência em imaginar uma outra perspectiva de vida para essas pessoas, que permanecem então cristalizadas no tempo, sendo as representações ancoradas no modelo do dano, a forma clássica de se operar, conferindo os obstáculos, muito maior força e importância do que nossa capacidade de enfrentá-los ${ }^{(23)}$.

\section{c) Representação de Transtorno Mental como Deficiência Mental}

As narrativas elaboradas pelos sujeitos revelam que há certa indefinição ou confusão entre os dois conceitos acima:
... eu vejo vários tipos de doentes mentais.... tem uns do- entes mentais que tem um.... que fez com que a pessoa tivesse tido uma deficiência mental grave. Deficiência é a mesma coisa? Doente ou Deficiente? (S3).
... eu não tenho muito contato com pessoa de saúde men- tal, embora tenha trabalhado na APAE (S3).

Revendo a história da psiquiatria, vemos muitas vezes que remanescentes dos antigos manicômios tiveram como diagnóstico a Oligofrenia, e, dadas as longas internações, foram segregados e afastados do convívio social e da família.

O acompanhamento junto a um núcleo familiar com um membro portador de deficiência mental demonstrou que o cuidado prestado pela família esteve sempre atado à internação psiquiátrica, isto é, o hospital psiquiátrico acolhia e protegia, ancorado em conceitos embasados em ideologias caritativas. Por outro lado, a família, carente de informação, confusa e, muitas vezes, cansada devido ao aumento dos encargos, sofria a falta de um serviço de referência e de apoio efetivo, que viesse realmente ajudar a lidar com os problemas vividos $^{(24)}$.

Desse modo, os discursos que mencionam a deficiência mental como sinônimo de transtorno mental refletem a realidade ainda presente nas instituições manicomiais que, ao longo do tempo, exerceram a tutela dos casos. 


\section{d) Representação de Transtorno Mental como Expressão de Intenso Sofrimento Psíquico}

O entendimento quanto ao transtorno mental permeado de muito sofrer psíquico, visto também na comunidade, está presente no relato:

\begin{abstract}
... acho que não é só aquele que está no Hospital Psiquiátrico, aqueles que estão em casa, na rua, no bairro, abrange um monte de coisa (S1).
\end{abstract}

\begin{abstract}
... eu acho complicado porque tem vários tipos, né? Tem a pessoa que sofre sozinha e não incomoda ninguém, que precisam muito, tem pessoas que estão mais agressivas, tem as que estão na rua e tem que ficar presas (S2).
\end{abstract}

Por outro lado, implica também a consideração de que a doença pode estar menos distante.

Em relação às concepções etiológicas sobre loucura, junto a profissionais de saúde, percebeu-se que há o enraizamento do entendimento biológico, pleno de abstrações e distanciamentos, tendo como resultado a concepção de que a loucura é algo que só pode ocorrer ao outro, tornando-se assunto alheio ${ }^{(25)}$.

O sofrer psíquico mais próximo da condição humana é mencionado:

Tem os que sofrem de depressão, tem uns aqui da área que sofrem, que por coisinhas meio que explodem... tem drogado, alcoolismo também (S1).

O relato denota a necessidade de compreensão desse panorama multifacetado que o sujeito vivencia na prática da assistência, num cenário de muitas dimensões inter-relacionadas, que envolvem movimentos e conflitos a serem enfrentados. Desse modo, o alcoolismo, as drogas, os transtornos mentais, a violência, são configurações que compõem os sistemas sociais em que os sujeitos são chamados a intervir.

\section{CONSIDERACÕ̃ES FINAIS}

Neste estudo, verificamos a internalização de conceitos ancorados em práticas tradicionais, que situam o transtorno mental como:

a) Imutável e socialmente excludente;

b) Loucura ou estado continuadamente irracional;

c) Incapacidade e impossibilidade de autonomia;

d) Deficiência mental.

Essas representações, além de criticáveis do ponto de vista da ciência atual, colocam obstáculos para o processo de transformação da assistência, na medida em que não consideram as potencialidades do portador de transtorno mental, deste modo eliminando a priori qualquer possibilidade de prática reabilitadora.

Notamos que apenas a última representação do transtorno mental identificada, como Intenso Sofrimento Psíquico, traz importante subsídio para a prática dos agentes comunitários, uma vez que revela compreensão do estado subjetivo do portador de transtorno, e abre a possibilidade de uma ação de assistência solidária, pautada na compreensão e na criação de vínculos afetivos.

As narrativas revelaram que a assistência em saúde mental tem implicações sociais e econômicas de difícil entendimento. Os sujeitos ouvidos demonstraram grande carência de informação, de capacitação para o enfrentamento das complexas situações presentes nos seus cotidianos de trabalho. Mostraram ainda carência de treinamento específico, que se caracterize por ser dinâmico e pautado na realidade vivenciada por cada comunidade, possibilitando o enfrentamento de conflitos presentes no exercício da assistência por esses profissionais.

No processo de efetivação do PSF e, com ele, de uma nova possibilidade de assistência integral à saúde, voltada às necessidades dos diferentes sujeitos, a assistência em Saúde Mental configura-se como real desafio. A equipe do PSF, em particular os agentes comunitários, precisa estar preparada para lidar com o sofrimento psíquico, cuidar das dificuldades sentidas e superá-las.

O cotidiano de trabalho dos agentes comunitário é permeado pelo recebimento de muitas solicitações advindas da comunidade. A identificação de suas representações sociais sobre o transtorno mental, realizada neste estudo, poderá contribuir para um melhor entendimento da atuação dos agentes e dos obstáculos por eles enfrentados. A partir de nosso estudo, pode-se fazer uma comparação das representações do transtorno mental dos agentes comunitários com as dos demais membros da equipe de saúde. À medida que se intensifica a proposta de uma assistência mais abrangente, há maior necessidade de eficiência do serviço, implicando uma mudança cultural envolvendo os profissionais de saúde, usuários dos programas de saúde, as famílias e toda a comunidade.

Ficou evidente a necessidade premente de investimento nos recursos humanos, promovendo o desenvolvimento de habilidades para lidar com as questões relacionadas à afetividade, à subjetividade, aos vínculos, para converter em realidade a desejada e efetiva assistência à saúde. Vemos ainda a necessidade de transformação da cultura manicomial e, nesse processo, efetivar a revisão de valores, de condutas, de princípios e de ética. 


\section{REFERÊNCIAS}

1. Rosa WAG. A contribuição da saúde mental para o desenvolvimento do Programa de Saúde da Família - PSF [dissertação]. Ribeirão Preto: Escola de Enfermagem de Ribeirão Preto, Universidade de São Paulo; 2002.

2. Pereira MAO, Pereira Junior A. Transtorno mental: dificuldades enfrentadas pela família. Rev Esc Enferm USP. 2003;37(4): 92-100.

3. Souza CR. Produção de sentidos por profissionais de saúde da família acerca do doente mental e dos cuidados a ele dirigidos [tese]. Ribeirão Preto: Escola de Enfermagem de Ribeirão Preto, Universidade de São Paulo; 2004.

4. Pereira MAO. A Reabilitação psicossocial no atendimento em saúde mental: estratégias em construção [tese livre-docência]. Ribeirão Preto: Escola de Enfermagem de Ribeirão Preto, Universidade de São Paulo; 2003.

5. Rotelli F, Leonardis O, Mauri D. Desinstitucionalização, uma outra via: a reforma psiquiátrica italiana no contexto da Europa Ocidental e dos "Países Avançados". In: Nicácio F, organizador. Desinstitucionalização. São Paulo: Hucitec; 2001. p. 17-59.

6. Lancetti A. saúde mental nas entranhas da metrópole. In: Jatene AD, Lancetti A, Mattos SAF. Saúde loucura: saúde mental e saúde família. São Paulo: Hucitec; 2000. p. 11-52.

7. Brêda MZ, Rosa WAG, Pereira MAO, Scatena MCM. Duas estratégias e desafios comuns: a reabilitação psicossocial e a saúde da família. Rev Lat Am Enferm. 2005;13(3):450-2.

8. Andrade L, Viana MC, Silveira CM. Epidemiologia dos transtornos psiquiátricos na mulher. Rev Psiquiatr Clin. 2006;33(2): 43-54.

9. Pereira MAO. Representação da doença mental pela família do paciente. Interface Comunic Saúde Educ. 2003;7(12):71-82.

10. Lunardelo SR. O trabalho do agente comunitário de saúde nos Núcleos de Saúde da Família em Ribeirão Preto [dissertação]. Ribeirão Preto: Escola de Enfermagem de Ribeirão Preto, Universidade de São Paulo; 2004.

11. Martines VRV. Compreendendo o processo de sofrimento no trabalho do agente comunitário de saúde no Programa Saúde da Família [dissertação]. São Paulo: Escola de Enfermagem, Universidade de São Paulo; 2005.

12. Franco L, Lancetti A. Quatro observações sobre as parcerias. In: Jatene AD, Lancetti A, Matto SAF. Saúde loucura: saúde mental e saúde família. São Paulo: Hucitec; 2000. p. 103-7.
13. Vaisberg TMA. Ser e fazer: enquadres diferenciados na clínica winnicottiana. Aparecida (SP): Idéias e Letras; 2004.

14. Laplanche J, Pontalis JB. Vocabulário de psicanálise. $2^{\mathrm{a}}$ ed. São Paulo: Martins Fontes; 1992.

15. Bardin L. Análise de conteúdo. Lisboa: Edições 70; 1977.

16. Moscovici S. A Representação social da psicanálise. Rio de Janeiro: Zahar; 1986.

17. Perrusi A. Imagens da loucura: representação da doença mental na psiquiatria. São Paulo: Cortez; 1995.

18. Machado MCL. Universo em desencanto: conceitos, imagens e fantasias de pacientes psiquiátricos sobre loucura e ou doença mental [tese]. São Paulo: Instituto de Psicologia, Universidade de São Paulo; 1995.

19. Minzoni MA. Assistência ao doente mental internado: análise de uma experiência de treinamento de atendentes em hospital psiquiátrico. Bol Oficina Sanit Panam. 1979;87(1): 50-9.

20. Gentile C. O Transtorno mental na percepção da equipe de enfermagem de hospital geral. [dissertação]. Ribeirão Preto: Escola de Enfermagem de Ribeirão Preto, Universidade de São Paulo; 2004.

21. Kinoshita RT. Contratualidade e reabilitação psicossocial. In: Pitta AM. Reabilitação psicossocial no Brasil. São Paulo: Hucitec; 1996 . p. 55-9.

22. Spivak M. Introduzione alla riabilitazione sociale: teoria, tecnologia e metodi di intervalo. Riv Sperim Freniatr. 1987; 106(3):522-74.

23. Valentini W. Nenhum ser humano será bonsai. In: Harari A, Valentini W, organizadores. A reforma psiquiátrica no cotidiano. São Paulo: Hucitec; 2001. p. 11-24.

24. Suzuki E. A visão da família em relação à internação psiquiátrica: um estudo de caso [monografia]. Ribeirão Preto: Curso de Especialização em Enfermagem Psiquiátrica, Escola de Enfermagem de Ribeirão Preto, Universidade de São Paulo; 2001.

25. Tsu TMA, Tofolo V. Concepções etiológicas de pacientes psiquiátricos sobre doença mental. Psicol USP. 1990;1(2):155-66. 